\title{
Singular quasiparticle scattering in the proximity of charge instabilities
}

\author{
C. Castellani, C. Di Castro, and M. Grilli \\ Dipartimento di Fisica, Università di Roma "La Sapienza", \\ Piazzale A. Moro 2, Roma, Italy 00185
}

We analyze the behavior of the dynamic scattering amplitude between Fermi liquid quasiparticles at the Fermi surface in the proximity of a charge instability, which may occur in the high temperature superconducting cuprates. Within the infinite- $\mathrm{U}$ Hubbard-Holstein model in the slave-boson large-N technique we find that, in the absence of long-range Coulomb forces the scattering amplitude is strongly singular at zero momentum transfer close to the phase separation instability and it has the same form provided by gauge-field theories. In the presence of long-range Coulomb forces the charge instability occurs at finite wavevectors and concomitantly the scattering is still singular but anisotropic. Nevertheless it remains strong over extended regions of the momentum space. In both cases we show how normal state properties are largely affected by this scattering.

PACS:74.72.-h, 71.27.+a, 72.10.-d

It is generally accepted that the understanding of the pairing mechanism in high $T_{c}$ superconductors is related to the understanding of the anomalous behaviour of the normal phase.

The anomalous properties of the normal phase have been interpreted along two distinct theoretical lines. One possible explanation is that the low dimensionality of these highly anisotropic systems and their correlated nature are at the origin of a breakdown of the Fermi liquid (FL).In particular the proposal of a Luttinger liquid formation in two dimension [1] has been intensively investigated [2]. The alternative aptitude has been to accept the Landau theory of normal FL's as a suitable starting point. The anomalous properties would then arise as a consequence of strong scattering processes at low energy between the quasiparticles. Along this line magnetic scattering has been considered to be responsible for both the anomalous properties of the normal phase and for the superconducting pairing [3]. Strong scattering may even lead to a complete disruption of the FL phase. In particular it was proposed that excitonic scattering could give rise to the so called marginal FL [4], and could also provide a pairing mechanism. Singular scattering can also be provided by gauge fields [5], which arise by implementing the resonating-valence-bond idea in the t-J model.

In this letter we want to understand whether phase separation (PS) or the incommensurate charge density wave (ICDW) instability are sources of strong scattering besides the above mechanisms. Indeed the complex nature of the phase diagram as a function of doping and temperature indicates that various energy scales of differ- ent nature (magnetic, excitonic,...) of the same order of magnitude compete to determine the low-energy physics and may lead to various instabilities, among which PS or charge instabilities may play a relevant role.

After PS was shown to be present in the phase diagram of the t-J model [6, ,7], we pointed out that PS commonly occurs in models with short range interaction [8 15], provided the strong local $e-e$ repulsion inhibits the stabilizing role of the kinetic energy. We therefore stressed that PS and superconductivity can be related phenomena irrespective of the nature of the short-range interaction. Emery and Kivelson [16] suggested that, although long range Coulomb (LRC) forces spoil PS as a static thermodynamic phenomenon, the frustrated tendency towards phase separation may still be important and give rise to large amplitude collective density fluctuations. Approaching the problem within a coarse-grained model, they suggested that these fluctuations may be responsible for the anomalous behaviour of the normal phase and for the superconducting pairing.

To assess the relevance of PS as a mechanism for anomalous scattering, we here determine the dynamical effective scattering interactions among quasiparticles close to a charge instability, both in the presence and in the absence of LRC forces. We carry out this analysis within a microscopic treatment of the Hubbard-Holstein model in the infinite- $U$ limit. We find that, both in the presence and in the absence of LRC forces, the dynamic effective interaction turns out to have a singular behaviour, strongly affecting the single-particle and the transport scattering time.

- The model - Although our results are quite generic of models with PS, to be specific we use as a simple paradigm the two-dimensional Hubbard model with an additional dispersionless phonon mode $A$ coupled $\grave{a} l a$ Holstein

$$
\begin{aligned}
& H=-t \sum_{\langle i, j\rangle, \sigma}\left(c_{i \sigma}^{\dagger} c_{j \sigma}+H . c .\right)-t^{\prime} \sum_{\langle\langle i, j\rangle\rangle, \sigma}\left(c_{i \sigma}^{\dagger} c_{j \sigma}+H . c .\right) \\
& +\omega_{0} \sum_{i} A_{i}^{\dagger} A_{i}+g \sum_{i, \sigma}\left(A_{i}^{\dagger}+A_{i}\right)\left(n_{i \sigma}-\left\langle n_{i \sigma}\right\rangle\right) \\
& -\mu_{0} \sum_{i \sigma} n_{i \sigma}+U \sum_{i} n_{i \uparrow} n_{i \downarrow}+\sum_{q}^{\prime} \frac{V_{C}}{\sqrt{G^{2}(q)-1}} \rho_{q} \rho_{-q}, \quad
\end{aligned}
$$

where $\langle i, j\rangle$ and $\langle\langle i, j\rangle\rangle$ indicate nearest-neighbor and next-nearest-neighbor sites respectively and $\sum_{\sigma} n_{i \sigma}=$ $\sum_{\sigma} c_{i \sigma}^{\dagger} c_{i \sigma}$ is the local electron density, which in momentum space is given by $\rho_{q} \equiv \sum_{k, \sigma} c_{k+q, \sigma}^{\dagger} c_{k, \sigma}$. The 
last term of Eq.(1) is a Coulombic potential between electrons on a two-dimensional square lattice (with lattice spacing $a$ in the $\mathrm{x}$ and $\mathrm{y}$ directions) embedded in a three-dimensional space with a separation $d$ between the planes in the $\mathrm{z}$ direction. The dielectric constants in the plane and perpendicular to it are $\epsilon_{\|}$and $\epsilon_{\perp}$ respectively and the Coulombic coupling constant $V_{C}=e^{2} d / 2 \epsilon_{\perp} a^{2}$. The momentum dependence of the potential on the $\mathrm{z}=0$ plane is found to be $G\left(q_{x}, q_{y}\right)=$ $\frac{\epsilon_{\|}}{\epsilon_{\perp}(a / d)^{2}}\left(\cos \left(a q_{x}\right)+\cos \left(a q_{y}\right)-2\right)-1$. As usual, the sum in the Coulombic potential does not include the zeromomentum component, since we are supposing that the diverging $q=0$ interaction between the electrons is canceled by the contribution of a uniform positively charged ionic background.

Since we are interested in strong local repulsion we take the limit $U \rightarrow \infty$, which gives rise to the local constraint of no double occupancy $\sum_{\sigma} n_{i \sigma} \leq 1$. To implement this constraint we use a standard slave-boson technique [17], by performing the usual substitution $c_{i \sigma}^{\dagger} \rightarrow c_{i \sigma}^{\dagger} b_{i}, \quad c_{i \sigma} \rightarrow$ $b_{i}^{\dagger} c_{i \sigma}$ and introducing a Lagrange multiplier field $\lambda_{i}$. The quartic Coulombic term can be decoupled by a HubbardStratonovich transformation introducing an additional real bosonic field $Y_{i}$. Within the large- $N$ expansion we assume that the spin index runs from 1 to $N$ and we relax the constraint to the form $\sum_{\sigma} c_{i \sigma}^{\dagger} c_{i \sigma}+b_{i}^{\dagger} b_{i}=\frac{N}{2}$. A suitable rescaling of the hoppings $t \rightarrow t / N$ and $t^{\prime} \rightarrow t^{\prime} / N$ must, in this model, be joined by the similar rescaling of the $e$-ph coupling $g \rightarrow g / \sqrt{N}$ and of the Coulomb interaction $V_{C} \rightarrow V_{C} / N$ in order to compensate for the presence of $N$ fermionic degrees of freedom.

The model can first be solved in the mean field $(N=$ $\infty)$ approximation by setting the $b_{i}$ and $\lambda_{i}$ bosons to their constant self-consistent values $b_{0}$ and $\lambda_{0}$ respectively. The system then results into free quasiparticles with a shifted chemical potential $\mu=\mu_{0}-\lambda_{0}$ and a dispersion $E_{k}=-2 t r_{0}^{2} \varepsilon_{k}$ with $\varepsilon_{k} \equiv\left(\cos \left(a k_{x}\right)+\cos \left(a k_{y}\right)\right)+$ $\left(t^{\prime} / t\right)\left(\cos \left(a k_{x}+a k_{y}\right)+\cos \left(a k_{x}-a k_{y}\right)\right)$ where $r_{0}^{2}=$ $b_{0}^{2} / N=\delta / 2$. For any finite doping $\delta$ at $\mathrm{T}=0$ the system is a Fermi-liquid where the mean-field value of the slave-boson field $b_{0}$ multiplicatively reduces the hoppings, $t \rightarrow t b_{0}^{2}, t^{\prime} \rightarrow t^{\prime} b_{0}^{2}$, thus enhancing the effective mass of the quasiparticles. At this level the mean-field self-energy does not introduce a finite quasiparticle lifetime.

The effective interaction leading to scattering between quasiparticles arises from the exchange of the bosonic fields in the $1 / N$ corrections beyond mean field approximation. One can define a four-component field $\alpha^{\mu}=$ $(\delta r, \delta \lambda, \phi, Y) . \phi_{i}=\left(A_{i}^{\dagger}+A_{i}\right) /(2 \sqrt{N})$ is the lattice displacement field, and $\delta r_{i}$ and $\delta \lambda_{i}$ are the fluctuating part of the $b_{i}$-field amplitude and of the the Lagrange multiplier respectively. The leading-order expressions of the effective scattering amplitude in the particle-hole channel can be written as
$\Gamma\left(k, k^{\prime} ; q, \omega\right)=-\sum_{\mu \nu} \Lambda^{\mu}\left(k^{\prime},-q\right) D^{\mu \nu}(q, \omega) \Lambda^{\nu}(k, q)$.

where $\Lambda^{\mu}$ are the vertices coupling the fermionic quasiparticles to the bosons, $\Lambda_{r}(k, q)=$ $-2 \operatorname{tr}_{0}^{2}\left(\varepsilon_{k+q / 2}+\varepsilon_{k-q / 2}\right), \Lambda_{\lambda}(k, q)=i, \Lambda_{\phi}(k, q)=-2 g$ and $\Lambda_{Y}(k, q)=i . D^{\mu \nu}(q, \omega)=\left\langle\alpha^{\mu}(q, \omega) \alpha^{\nu}(-q,-\omega)\right\rangle=$ $N^{-1}(2 B+\Pi(q, \omega))_{\mu \nu}^{-1}$ is the leading order boson propagator with self-energy corrections given by the fermionic bubbles $\Pi^{\mu \nu}$ (which include quasiparticle-boson vertices). $B$ is the bare boson-propagator matrix. An expression similar to Eq. (2) holds for the scattering amplitude in the particle-particle channel.

- The results - The evaluation of the density correlation function $P(q, \omega) \equiv(1 / N) \sum_{\sigma \sigma^{\prime}}\left\langle n_{\sigma}(q, \omega) n_{\sigma^{\prime}}(-q,-\omega)\right\rangle$ provides information on the stability of the system. In particular a divergence in the static density-density correlation function $P(q, \omega=0)$ signals the occurrence of PS (at $q \rightarrow 0$ ) or of CDW instabilities (at finite $q$ 's). A complete investigation of the static and dynamical properties of the present model together with the analysis of its stability was already carried out in a previous work [15]. Here we just mention that, within the present formalism, the model (1) displays a phonon-driven charge instability even for rather small e-ph coupling. In the absence of LRC forces this instability occurs before any other finite $q$ instability up to intermediate-large doping. At large doping the instability requires larger e-ph couplings and occurs at finite $q \approx 2 k_{F}$ in some directions [in particular $(1,0)$ and $(0,1)$ ] signalling the occurrence of incommensurate CDW. The introduction of LRC forces eliminates the small- $q$ divergence in the static correlation function always giving rise to finite $q$ instabilities. The critical $q$, in this case, is not related to any pseudonesting of the Fermi surface but it depends on the strength of the LRC forces and on the momentum dependence of the poles in the divergent static correlation function with only short-range forces. Pair formation is always found near the instabilities.

As already announced, we want to report on the behavior of the quasiparticle scattering close to both the PS and the CDW instability. It is worth noting that a divergent scattering amplitude will follow from a divergent correlation function $P$. Indeed a divergent boson propagator enters in the expression of both quantities establishing a clear connection between the charge instability and the singular quasiparticle scattering.

In this regard we carried out an extensive analysis of the real and imaginary parts of the scattering amplitude between the quasiparticles on the Fermi surface.

As expected, near the PS instability $\left(V_{C}=0\right)$, the anomalous behavior of $\Gamma$ is identified to be of the form

$$
\Gamma(q, \omega) \approx-\frac{1}{B q^{2}-i \omega \frac{C}{q}+D}
$$

As shown in Fig. 1a, for the model with $t=0.5 \mathrm{eV}$, $t^{\prime} / t=-1 / 6, \omega_{0}=0.04 \mathrm{eV}$ and $g=0.194 \mathrm{eV}, D=D(\delta-$ 
$\delta_{c}$ ) vanishes linearly when, for a given $g$, the instability takes place at the critical doping $\delta_{c}=\delta_{c}(g)$. For the same values of the parameters, Fig.1b reports the real static scattering amplitude $\Gamma(q, \omega=0)$ as a function of transferred momentum $q$ at various dopings close to the $q=0$ instability. In this case the singular part of the scattering amplitude displays a quite isotropic behavior (at $\left.q_{c}=0\right)$.

In the absence of LRC forces the singular behavior of $\Gamma(q \rightarrow 0, \omega=0)$ at the PS instability is by no means surprising within a FL framework. Indeed, the FL expression for the compressibility is $\kappa=2 \nu^{*} /\left(1+2 \nu^{*} \Gamma_{\omega}\right)=$ $2 \nu^{*}\left(1-2 \nu^{*} \Gamma_{q}\right)$, where $\Gamma_{\omega}$ and $\Gamma_{q}$ are the standard dynamic $(\omega \rightarrow 0, q=0)$ and static $(q \rightarrow 0, \omega=0)$ limit of the scattering amplitude. This indicates that a divergent $\kappa$, when the quasiparticle mass remains finite $\left(\nu^{*}<\infty\right)$, only happens when $F_{0}^{s} \equiv 2 \nu^{*} \Gamma_{\omega} \rightarrow-1$ (Pomeranchuk criterion). At the same time if $\Gamma_{q} \rightarrow \infty$. We like to point out here that the above arguments keep their full validity irrespective of the mechanism leading to PS.

In the presence of LRC forces the singular part of $\Gamma$ can be written as

$$
\Gamma(\boldsymbol{q}, \omega) \approx-\frac{A}{\omega_{\boldsymbol{q}}-i \omega}
$$

where $\omega_{\boldsymbol{q}}=D^{\prime}+B^{\prime}\left|\boldsymbol{q}-\boldsymbol{q}_{c}\right|^{2}$ The behavior of the mass term $D^{\prime}$ is shown in Fig. 2a for the model with $t=0.5 \mathrm{eV}, t^{\prime} / t=-1 / 6, V_{C}=1.1 \mathrm{eV}, \omega_{0}=0.04 \mathrm{eV}$ and $g=0.240 \mathrm{eV}$ as a function of $\delta-\delta_{c}$. For these parameters the instability first occurs at $\delta_{c}=0.194$ and $\boldsymbol{q}_{c} \approx( \pm 0.28 / a, \pm 0.86 / a)$, or $\boldsymbol{q}_{c} \approx( \pm 0.86 / a, \pm 0.28 / a)$. Analogously to Fig. 1b, Fig. 2b displays the strong doping dependence of the static scattering amplitude as a function of momenta in the $\boldsymbol{q}_{c}$ direction. However, as shown in Fig. 3, the scattering is quite strong, although non-singular, in all directions for $|\boldsymbol{q}| \approx\left|\boldsymbol{q}_{c}\right|$. We also checked that the (almost) isotropic contribution to the static scattering amplitude it is much less fragile under doping variations.

The imaginary term in the denominators in the r.h.s. of Eqs.(3) and (4) reproduces on a wide range of transferred momenta $q$ the behaviour of the imaginary part of the mean field fermionic polarization bubble $\operatorname{Im}\left[P^{0}(\boldsymbol{q}, \omega)\right] \propto \omega / q$ at small $\omega$. This indicates, that, despite the complicated formal structure of the scattering amplitude (2) arising from the matrix form of the boson propagator $D^{\mu \nu}$, near the instability a simple RPA-like structure results in the final expression. The forms (3) and (4) are, therefore, generic of PS or charge instabilities.

It is apparent that the effective interaction (3) in the isotropic pure-short-range case, has the same form as the one mediated by gauge fields [5], if it were not for the doping-dependent mass term and for the completely different origin of the singularity. The LRC forces make the scattering anisotropic rendering it more similar to the magnetic fluctuation case [3], even though the really singular behaviour is for differently oriented momenta and large scattering is present in any direction.

Near PS at $\delta=\delta_{c}$, the electrons are coupled to a mode, which is soft at long wavelength, and the same results of Ref. [5] apply. Therefore in two dimensions the inverse scattering time is proportional to $T^{2 / 3}$ and the resistivity $\rho$ is proportional to $T^{4 / 3}$ with a crossover temperature $T^{*}$ to the standard FL behavior, which in our case turns out to be $T^{*} \propto\left(\delta-\delta_{c}\right)^{3 / 2}$. The resistivity is anomalous but it is not linear in $\mathrm{T}$ as in the optimally doped high $T_{c}$ materials. An additional the limitation of the pure short-range case may be the difficulty in approaching the instability line which might be embedded in a region stabilized by the Maxwell construction.

In the more physical case including LRC forces, the proposal of associating the anomalous properties of the normal phase to the presence of a $T=0$ quantum critical point [19,20] has a natural realization within the chargeinstability mechanism. The outcome for the transport properties should be similar to the one described within the nearly antiferromagnetic FL scenario [3], i.e. a linear resistivity for temperatures larger than a crossover temperature $T^{*}$, which, in our case would be proportional to $D^{\prime}$ in Eq.(4). However, this result was questioned in Ref. 21] for the AF fluctuations because only few "hot" points on the Fermi surface are connected by singular interactions, with an anomalous inverse scattering time proportional to $\sqrt{T}$. Generically the resistivity would be dominated by an inverse scattering time proportional to $T^{2}$, then linear resistivity would only appear for $T \gg T^{*}$. In our case, instead, a strong rather isotropic scattering persists rendering the above limitation less effective.

The above scenario connecting charge instabilities to the anomalous normal properties of the superconducting cuprates raises the problem of the relevance of charge fluctuations in these systems 22]. Indeed commensurate CDW have been recently observed 23 in a related compound $\left(\mathrm{La}_{2-x-y} \mathrm{Nd}_{y} \mathrm{Sr}_{x} \mathrm{CuO}_{4}\right.$ with $x=0.12$ and $\left.y=0.4\right)$ possibly due to the pinning of electronic dynamical CDW by a suitable underlying lattice structure. It is therefore quite important to assess the electronic origin of this CDW, e.g. by detecting non-linear effects like electricfield-induced depinning. This would strongly support the existence of dynamical CDW fluctuations when pinning is not effective leaving the materials in the metallic phase.

[1] P. W. Anderson, Science 235, 1196 (1987); Phys. Rev. Lett. 64, 1839 (1990); ibid. 65, 2306 (1990).

[2] For a review see The physics and the mathematical physics of the Hubbard model, edited by D. Campbell, 
Plenum Press 1995.

[3] P. Montoux and D. Pines, Phys. Rev. B50, 16015 (1994).

[4] C. M. Varma, Int. J. of Mod. Phys. B3, 2083 (1989).

[5] N. Nagaosa and P. A. Lee, Phys. Rev. Lett 64, 2450 (1990); P. A. Lee and N. Nagaosa, Phys. Rev. B46, 5621 (1992).

[6] V. J. Emery, S. A. Kivelson, and H. Q. Lin, Phys. Rev. Lett. 64, 475 (1990).

[7] M. Marder, N. Papanicolau and G. C. Psaltakis Phys. Rev. B 41, 6920 (1990).

[8] M. Grilli, R. Raimondi, C. Castellani, C. Di Castro, and G. Kotliar, Phys. Rev. Lett. 67, 259 (1991); Int. J. of Mod. Phys. B Vol. 5, 309 (1991).

[9] N. Cancrini, S. Caprara, C. Castellani, C. Di Castro, M. Grilli and R. Raimondi, Europhys. Lett. 14, 597 (1991).

[10] C. Di Castro and M. Grilli, Phys. Scr. T45, 81 (1992).

[11] R. Raimondi, C. Castellani, M. Grilli, Y. Bang, and G. Kotliar, Phys. Rev. B 47, 3331 (1993).

[12] C. Castellani, M. Grilli, and G. Kotliar, Phys. Rev. B 43, 8000 (1991); S. Caprara and M. Grilli, Phys. Rev. B 49, 697 (1994).

[13] S. Caprara, C. Di Castro and M. Grilli, Phys. Rev. B 51, $9286(1995)$.

[14] M. Grilli and C. Castellani, Phys. Rev. B 50, 16880 (1994).

[15] F. Becca, M. Tarquini, M. Grilli, C. Castellani, and C. Di Castro, preprint.

[16] V. J. Emery, and S. A. Kivelson, Physica C 209, 597 (1993); U. Löw, V. J. Emery, K. Fabricius, and S. A. Kivelson, Phys. Rev. Lett. 72, 1918 (1994).

[17] S. E. Barnes, J. Phys. F 6, 1375 (1976); P. Coleman, Phys. Rev. B 29, 3035 (1984); N. Read, and D. M. Newns, J. Phys. C 16, 3273 (1983).

[18] With this value for $V_{C}$ electrons on neighboring cells feel a repulsion of $\sim 0.2 \mathrm{eV}$ when $\epsilon_{\|}=5$ and $\epsilon_{\perp}=25$ and $d=$ $13 \mathrm{~A}$. These values are rather typical for $\mathrm{La}_{2-\mathrm{x}} \mathrm{Sr}_{\mathrm{x}} \mathrm{CuO}_{4}$ compounds.

[19] A. Sokol and D. Pines, Phys. Rev. Lett. 71, 2813 (1993).

[20] C. M. Varma, Phys. Rev. Lett. XX, XXXX (1995).

[21] R. Hlubina and T. M. Rice, Phys. Rev. B 51, 9253 (1995).

[22] Proceedings of the Workshop on Phase separation in cuprate superconductors, 6-12 May 1992, Erice, Italy, Eds. K.A. Müller and G. Benedek, World Scientific 1993; Proceedings of the Workshop on Phase separation, electronic inhomogeneities and related mechanisms for high $T_{c}$ superconductors, 9-15 July 1995, Erice, Italy.

[23] J. M. Tranquada, B. J. Sternlieb, J. D. Axe, Y. Nakamura and S. Uchida, submitted to Nature.
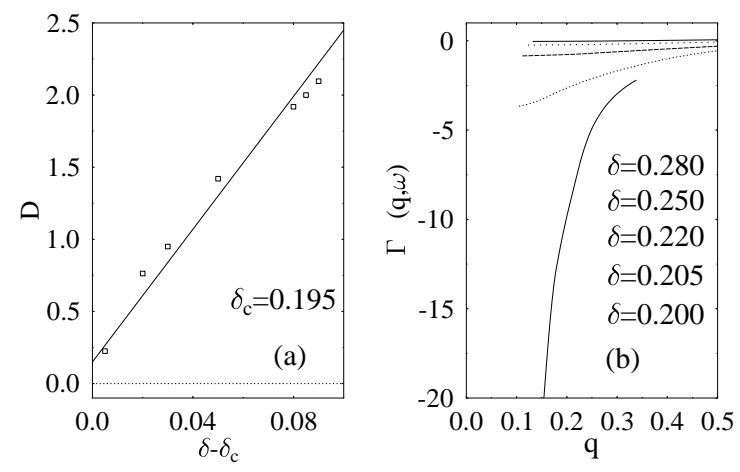

FIG. 1. (a) Mass $D$ as a function $\delta-\delta_{c}$ for $t=0.5 \mathrm{eV}$ $t^{\prime}=-1 / 6 t, V_{C}=0, \omega_{0}=0.04 \mathrm{eV}$ and $g=0.194 \mathrm{eV}$. (b) Static scattering amplitude for the same parameters as in (a) as a function of the transferred momentum $\boldsymbol{q}$ in the (1,0) direction. The doping $\delta=0.2,0.205,0.22,0.25,0.28$ increases from the lower solid line to the upper solid line.
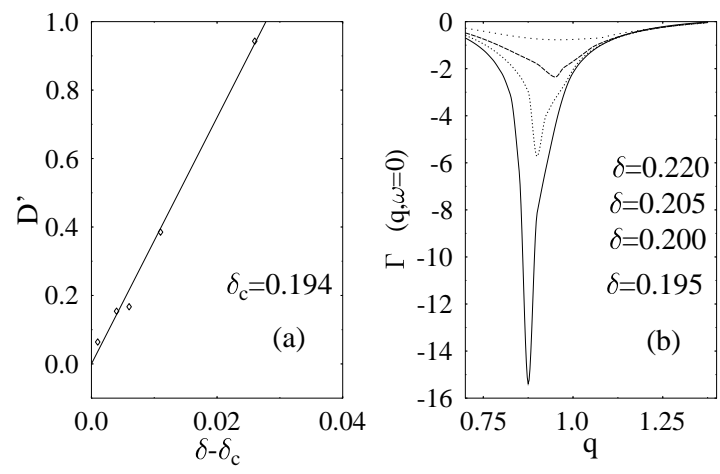

FIG. 2. (a) Mass $D^{\prime}$ as a function $\delta-\delta_{c}$ for $t=0.5 \mathrm{eV}$ $t^{\prime}=-1 / 6 t, V_{C}=1.1 \mathrm{eV}, \omega_{0}=0.04 \mathrm{eV}$ and $g=0.312 \mathrm{eV}$. (b) Static scattering amplitude for the same parameters as in (a) as a function of the transferred momentum $\boldsymbol{q}$ in the $\boldsymbol{q}_{c} \approx( \pm 0.28 / a, \pm 0.86 / a)$, direction. The doping $\delta=0.195,0.2,0.205,0.22$ increases from the lower solid line to the upper solid line. 


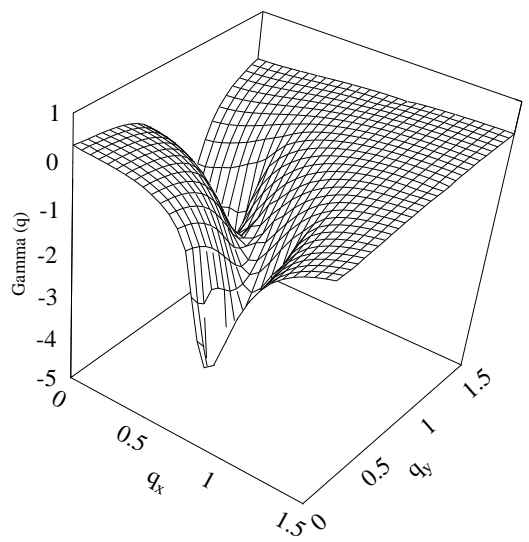

FIG. 3. Momentum dependence of the static scattering amplitude for the same parameters as in Fig. 2a at $\delta=0.195$. 